\title{
ANÁLISIS SOBRE EL SISTEMA DE VALOR DE LA PRODUCCIÓN SOJERA EN LA PROVINCIA DEL CHACO
}

CARLOS HAROLD GABRIEL LUCAS

FACULTAD DE CIENCIAS ECONÓMICAS UNIVERSIDAD NACIONAL DEL NORDESTE

ARGENTINA

"Nada puede ensanchar más tu mente que la habilidad de investigar sistemática y sinceramente todo lo que la vida ofrece a tu observación". Marco Aurelio. 


\section{RESUMEN}

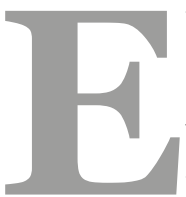

l enfoque de clúster refiere a una agrupación de organizaciones productivas que poseen caracteres comunes y ejercen de hecho ciertas acciones conjuntas. El sistema de valor de la soja generó en 2011 el 64\% del valor bruto de producción nacional de cultivos y explicó el $47 \%$ del producto bruto geográfico chaqueño. Este trabajo describe las características del sistema de valor de la producción sojera del Chaco con el fin de determinar el beneficio que representaría la instalación de un clúster. Es un estudio descriptivo, exploratorio y predominantemente cualitativo con datos de fuentes secundarias y bibliográficas. El Chaco se caracteriza por un sistema de valor incompleto dado que existen eslabones ausentes o débiles. La etapa primaria contiene 16 semilleros especializados, 4 fábricas de maquinarias agrícolas y un sector de fertilizantes. La producción en campo y el acopio se concentran en la zona del oeste Chaqueño. La etapa secundaria, un eslabón débil, comprende dos grados de industrialización en origen, el primero incluye 1 molinera, 1 procesadora de aceite y 2 de pellets; el segundo 1 planta de mezclado de biodiesel, sin producción farmacéutica ni alimenticia. La etapa de comercialización, abastece principalmente al mercado externo. En 2011 el 29,38\% de las exportaciones correspondía al complejo sojero, mayormente de poroto. En conclusión, siendo la etapa primaria decisiva en la economía chaqueña es recomendable potenciar su competitividad mediante políticas públicas como también la reactivación y sostenimiento del puerto de Barranqueras. Además, dada la fortaleza del sistema productivo argentino, el Chaco está en condiciones de replicarlo impulsando explotaciones sojeras sustentables cohesionadas en un clúster productivo.

Palabras clave: desarrollo rural estratégico, economía regional, clúster, aglomeraciones productivas, soja.

\section{INTRODUCCIÓN}

Mitnik (2011) señala que las economías de aglomeración datan de la época de la primera revolución industrial en Inglaterra, este fenómeno fue estudiado formalmente por Alfred Marshall quien publicó el resultado de sus observaciones en su obra clásica Principios de Economía. Sesenta años después, el economista Albert Hirschman (1961) planteó que las empresas se ven afectadas por dos efectos denominados "de eslabonamiento" (anterior y posterior).

Retomando ambas ideas, entre 1980 y 1990, el profesor Michael Porter presentó junto a treinta investigadores, su teoría sobre clústeres y sectores industriales basados en las ventajas competitivas plasmada en su libro: La ventaja competitiva de las naciones. Su enfoque innovador dejó atrás a la geografía y a la economía como marco del estudio de las aglomeraciones productivas y llevó a los clústeres al ámbito de la administración. De esa manera formuló los 
conceptos de cadena y sistema de valor.

A principios del siglo XXI, la escuela PENSA de la Universidad de Sao Pablo adecuó los conocimientos previos al ámbito de la estructura productiva agrícola propiciando el concepto de sistema agroalimentario como un conjunto de relaciones contractuales entre empresas y agentes especializados, cuyo objetivo final es el de captar al consumidor de un determinado producto (Morales, 2012).

Michael Porter logró realizar un análisis integrado entre los niveles empresa (micro) y sector (macro), con la interrelación de los conceptos de cadena y sistema de valor, respectivamente. La cadena de valor es definida por el mismo autor (citado por Morales, 2012), desde una óptica empresarial, como el conjunto de actividades estratégicas que deben ser analizadas para entender el comportamiento de los costos y las fuentes de financiación para entregar y apoyar a sus productos.

El sistema de valor es definido desde una óptica sectorial y agregada como el conjunto de cadenas de valor que se relacionan para llegar a producir un determinado valor para el cliente final. Es decir, incluye a proveedores y clientes de diversas especialidades articuladas por su participación en un bien o servicio específico. En el lenguaje técnico más tradicional suelen denominarse complejos, y éste es el término más utilizado en los ámbitos de decisión política.

Fue también Porter (1998), quien abocado a la tarea del análisis del éxito de los sectores industriales, concibió el concepto de clúster como un grupo de empresas interconectadas, geográficamente próximas e instituciones asociadas en un campo en particular, vinculadas por comunidades y complementariedades. El mismo ha sido objeto de controversias sobre su extensión y contenido, lo que llevó a que fuera evolucionando conforme se realizaban estudios empíricos.

En este trabajo se entiende por clúster a un modelo donde existe una agrupación de organizaciones productivas que poseen caracteres comunes y ejercen de hecho ciertas acciones conjuntas. Aplicado al contexto agrícola, la condición geográfica subyacente adquiere un rol fundamental, dado que la producción requiere de la explotación económica de tierras, que se convierten en su recurso crítico.

\section{OBJETIVOS Y METODOLOGÍA}

Entonces, es necesario cuestionarse cuáles son los rasgos salientes del sistema de valor de la producción sojera provincial, a fin de relacionarlo con el desarrollo de la teoría de clústeres.

Este trabajo tiene por objetivo caracterizar el sistema de valor de la producción sojera del Chaco con el fin de determinar cualitativamente el beneficio que representaría en dicho territorio la instalación de un clúster.

Las organizaciones productivas componentes del sistema de valor sojero se encontrarían presentes en la Provincia del Chaco; sin embargo, del relevamiento bibliográfico efectuado no 
se han hallado las características del mismo, ni su posible relación con el desarrollo teórico de clusterización, por lo que es relevante proceder a su estudio. Asimismo, se presume que es relacionable con la dicha temática.

El diseño metodológico responde a una investigación no experimental, de carácter exploratorio, dado que permite perfilar las variables que intervienen en el modelo a proponer, como mejor supuesto de reproducción de las relaciones existentes en la matriz de datos empíricos. Si se considera el tipo de información y el modo de recogerla, este estudio es de corte descriptivo mediante la técnica documental. A su vez, si se tiene en cuenta cómo se recolectan y analizan los datos, se está frente a una investigación bibliográfica de corte transversal.

Este trabajo se basa en fuentes secundarias, las más significativas provienen de estadísticas nacionales, provinciales y departamentales (superficie cosechada, producción, exportaciones, entre otras) como así también la sistematización de los aportes teóricos del campo económico y de la administración estratégica para aplicarlos al contexto provincial.

\section{EVOLUCIÓN Y CARACTERÍSTICAS DEL SISTEMA DE VALOR DE LA SOJA EN ARGENTINA}

Como premisa, un sistema de valor local o sub-nacional no debería ser analizado aisladamente del sistema nacional, si éste existiera. A continuación se describen las evidencias que señalan la evolución y características del mismo.

Desde fines del siglo XIX, Argentina había adoptado un modelo agro-exportador basado en cultivos tradicionales como el trigo, maíz y girasol. Concomitantemente, desarrolló un complejo cárnico que, hacia mediados del siglo XX, la posicionó como primer exportador mundial de carne bovina con un importante mercado interno (Rapoport, 2008). Sin embargo, a partir de la década de 1970 comenzó un proceso de transición donde, en solo 20 años, la soja pasó de ser un cultivo experimental (Martínez Dougnac, s.f.) a pelear el liderazgo con el maíz y el trigo.

Luego de la introducción de semillas genéticamente modificadas en la campaña 1996/97, la exportación del cultivo se convirtió en parte fundamental del "milagro argentino" al generar una salida a la crisis de 2001-2002, que había dejado al país con un índice de pobreza mayor al 50\% y un nivel de desocupación superior al 30\% (Struzzenegger, 2013). Hacia 2011, el mismo sistema generó el $64 \%$ del valor bruto de producción nacional de cultivos medido en dólares (Cristina \& Bulgarelli, 2013).

Es posible afirmar que el sistema de valor nacional de la soja se compone de tres etapas: primaria, secundaria (de industrialización) y de comercialización (Mas, 1998; Galeano, 2008; Giancola, Salvador, Covacevich e Iturrioz, 2009), cuya morfología puede observarse en la Figura 1. 


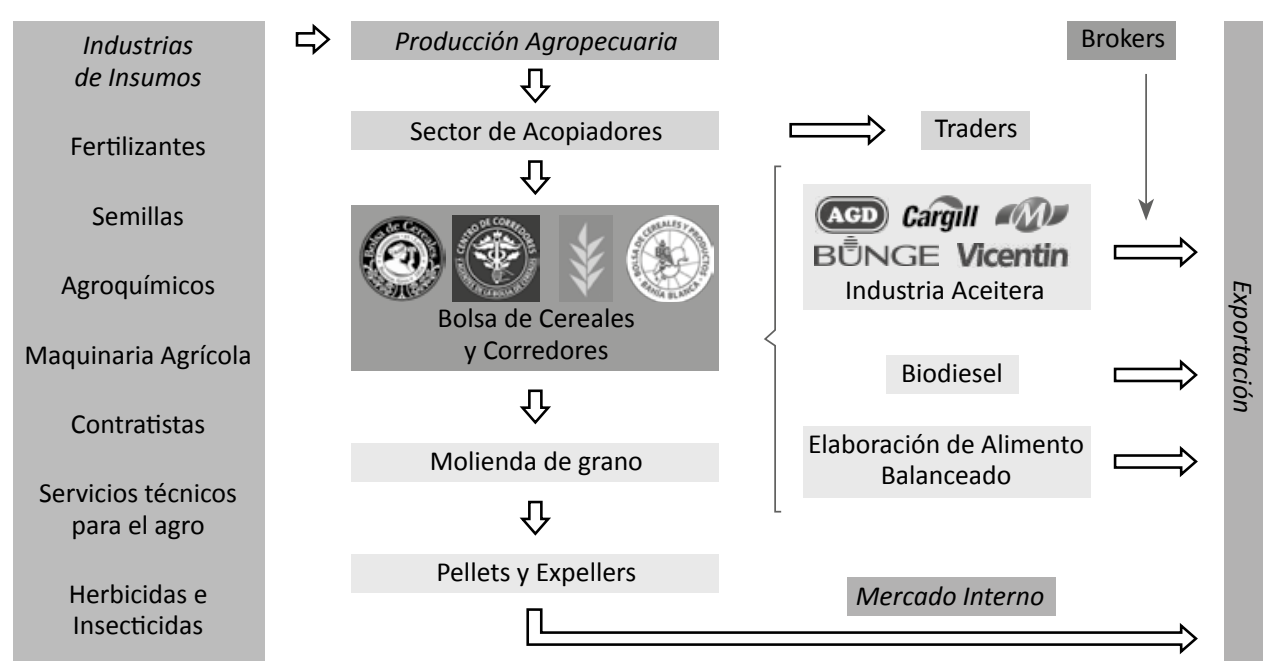

Figura 1. Representación Morfológica del sistema de valor nacional de la soja en Argentina (Elaboración propia)

La etapa primaria incluye la provisión de insumos, producción en campo y acopio. La provisión de insumos para la producción sojera se distribuye entre varias industrias (semillas, agroquímicos, fertilizantes, maquinaria agrícola) y el aporte de profesiones liberales para servicios técnicos (agrónomos, contadores, administradores rurales, abogados y otros especialistas).

La producción en campo requiere determinadas condiciones de aptitud del suelo y clima. La adopción de semillas genéticamente modificadas ha flexibilizado ambos requerimientos al crearse semillas adaptables a diversas zonas, predominando variedades propicias para el este argentino.

Luego de la producción agrícola, los granos deben ser almacenados y acondicionados para su posterior uso, dando lugar a la actividad de acopio (Castagna, Pellegrini \& Woelflin, 2011).

En una segunda etapa se produce una industrialización intermedia, que consiste en la molinería y producción de aceites (en bruto y refinados). Ambas ramas de la industria se encuentran concentradas al sur de la provincia de Santa Fe, especialmente la aceitera. Ésta es estructuralmente exportadora, altamente competitiva y de fuerte impacto en las economías regionales. Los bienes de la etapa intermedia nutren a la de industrialización final, en la que se obtienen biodiesel, fármacos y alimentos.

La producción de las distintas etapas sirve a dos mercados: nacional e internacional. Según datos de la Bolsa de Comercio de Rosario, Argentina exporta anualmente alrededor del 96\% de su producción de soja en forma de habas, aceite, harina y pellets de soja; y las estadísticas recolectadas por el International Trade Center permiten afirmar que el país ocupa la primera posición mundial como exportador de aceite y biodiesel. 


\section{EVOLUCIÓN Y CARACTERIZACIÓN DEL SISTEMA DE VALOR SOJERO EN LA PROVINCIA DEL CHACO}

En ese marco, la inserción del Chaco en la producción del cultivo fue marginal hasta la campaña 1996/97, la cual marcó un punto de inflexión en la estructura productiva agrícola de la provincia, propiciando el reemplazo del tradicional cultivo de algodón.

El cultivo de la oleaginosa fue creciendo progresivamente hasta explicar el $47 \%$ del producto bruto geográfico chaqueño, conforme a los últimos datos disponibles de 2011 (Capitanich, 2011).

Como puede observarse en la Figura 2, la participación de la soja en la producción agrícola, en términos de superficie cosechada, ha crecido aceleradamente. Además, desde 2009 se ha convertido en una importante fuente de recursos para la hacienda pública provincial por el carácter coparticipable de las retenciones aplicadas sobre la oleaginosa.

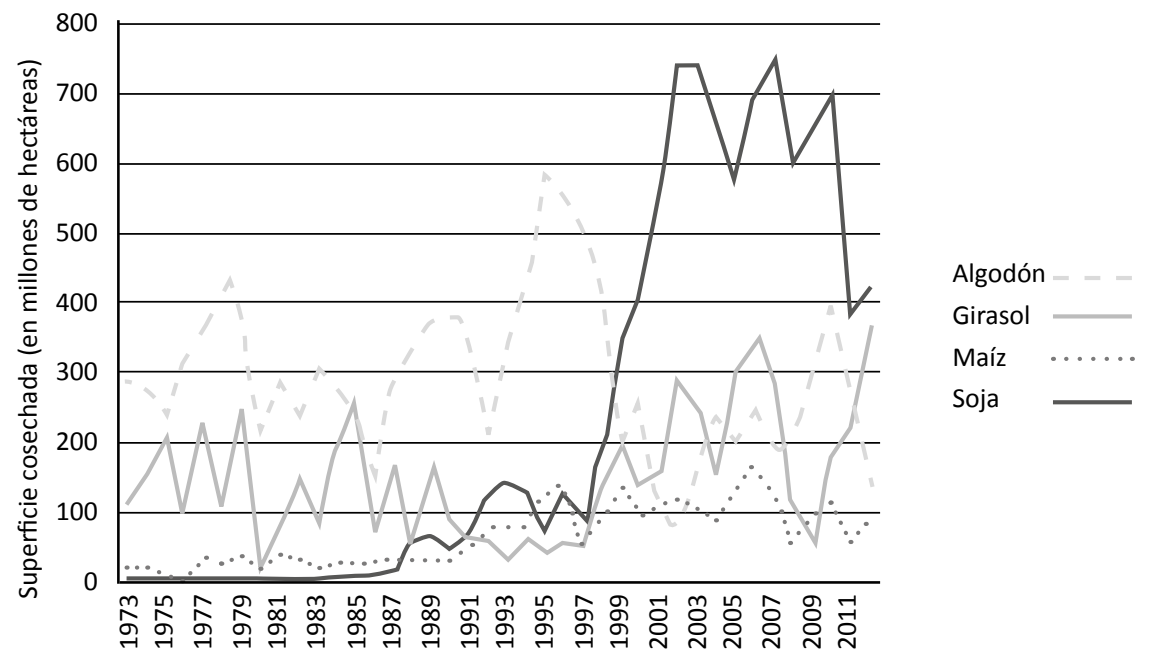

Figura 2. Evolución de la superficie cosechada de los 4 principales cultivos de la Provincia del Chaco entre las campañas 1973 y 2012, en miles de hectáreas (Elaboración propia en base a datos del Ministerio de Agricultura, Ganadería y Pesca de la Nación)

La provincia del Chaco se caracteriza por un sistema de valor incompleto dado que existen eslabones ausentes o débiles. Como se observa en la Figura 3, la fortaleza de los eslabones disminuye progresivamente a medida que se avanza hacia las etapas de mayor valor agregado.

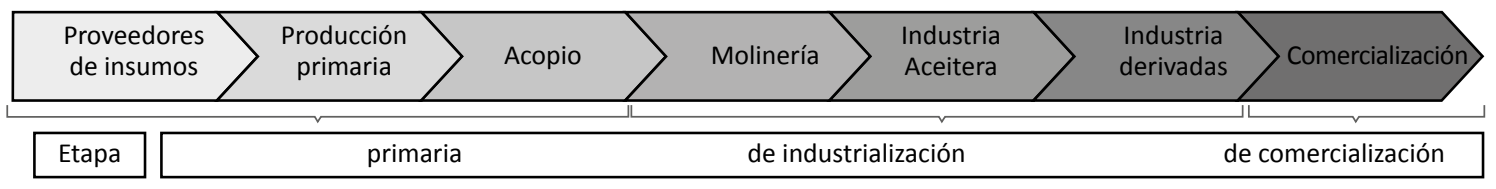

Figura 3. Representación simplificada del sistema de valor de la producción de soja en la Provincia del Chaco (Elaboración propia en base a datos del Ministerio de Agricultura, Ganadería y Pesca de la Nación) 
En la Figura 3, los colores más claros indican una menor presencia de actores.

La provisión de insumos perteneciente a la etapa primaria contiene 16 semilleros especializados federados en la Cámara Argentina de Semilleros Multiplicadores, una industria de maquinarias agrícolas en ascenso compuesta por 4 fábricas y un sector de fertilizantes dedicado exclusivamente a la comercialización.

La producción en campo se concentra en los departamentos: O'Higgins, General Belgrano, 9 de Julio, Chacabuco y 12 de Octubre, área que aportó el 58,5\% de la producción provincial en la campaña 2011/12 y el 57\% en la siguiente (véase Figura 4). El acopio está atomizado en la misma área geográfica y existe una federación, con sede en la ciudad de Las Breñas, que articula a 27 empresas del rubro.

La participación del Chaco en la producción nacional de la oleaginosa en la campaña 2012/13 ascendió al 1,12\% lo que la convierte en la provincia con mayor contribución de la región NEA.

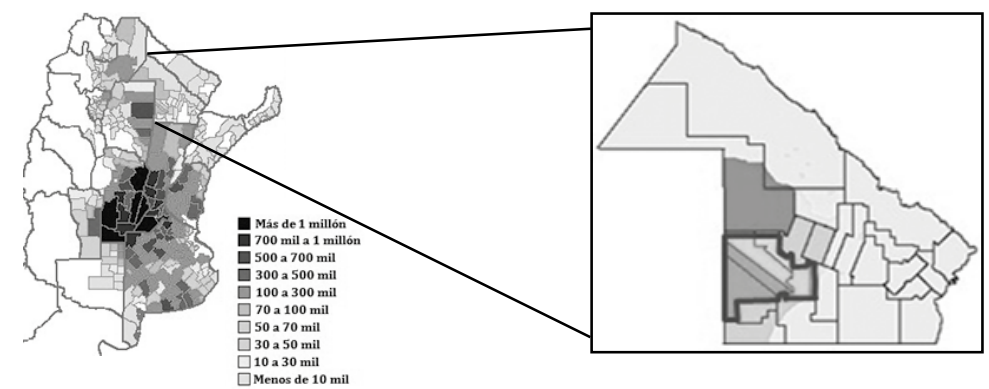

Figura 4. Zonas de producción de soja en Argentina categorizado por cantidad de toneladas producidas en la campaña 2012/13 con ampliación de la Provincia del Chaco para la misma campaña (Elaboración propia en base a datos del Sistema Integrado de Información Agropecuaria del Ministerio de Agricultura, Ganadería y Pesca de la Nación, Consejo Nacional de Investigaciones Científicas y Técnicas y Agencias de desarrollo territorial del Ministerio de la Producción de la Provincia del Chaco).

La etapa secundaria es uno de los eslabones débiles del sistema, comprendiendo dos grados de industrialización en origen. En el primero se hallan una planta procesadora de aceite y dos de pellet de soja junto a una molinera que contribuye con menos del 1\% de la producción nacional de la campaña 2011/12, según los últimos datos disponibles en CIARA; en el segundo se encuentra solo una planta de mezclado de gasoil con biodiesel, que además, es la única en la región del NEA, estando ausentes los sectores farmacéutico y alimenticio.

En la etapa de comercialización, lo producido en la provincia es captado principalmente por los mercados internacionales (cuyos principales clientes son China, Brasil, Chile y Colombia) el resto lo absorbe el mercado interno. Debe destacarse que el 29,38\% de las exportaciones chaqueñas en 2011 correspondió al complejo sojero, mayormente de poroto (CAC, 2013). La provincia contribuyó en el mismo año con el 3,39\% del total de las toneladas exportadas de habas de soja.

Lo descripto precedentemente permite apreciar las características del sistema de valor sojero chaqueño en inescindible relación con el que se presenta a nivel nacional. 


\section{DISCUSIÓN Y CONCLUSIONES}

En base a lo reseñado, la etapa más fortalecida del mismo es la primaria y su importancia para la economía chaqueña es decisiva. Por lo tanto es recomendable potenciar su competitividad a través de políticas públicas, tales como las que han propiciado la radicación de industrias de la siguiente etapa, pero sin atraer a las alimenticias y farmacéuticas. Cabe aclarar que, dada la importancia que posee la infraestructura logística para la actividad, la reactivación y sostenimiento del puerto de Barranqueras es un paso importante hacia esa mejora competitiva.

La relación que existe entre los conceptos de sistema de valor y clúster, puede asumirse como de complementación, dado que, un sistema de valor se compone de las cadenas de valor (que pueden ser agrupados en sectores) que trabajan para la generación de valor destinado a un cliente final, mientras que el clúster comprende total o parcialmente a las organizaciones productivas de un sistema de valor y las relaciona con otros actores de interés como los organismos públicos, de educación (media y superior), el sector financiero y los medios de comunicación. En la Figura 5 la intersección representa la relación conceptual señalada.

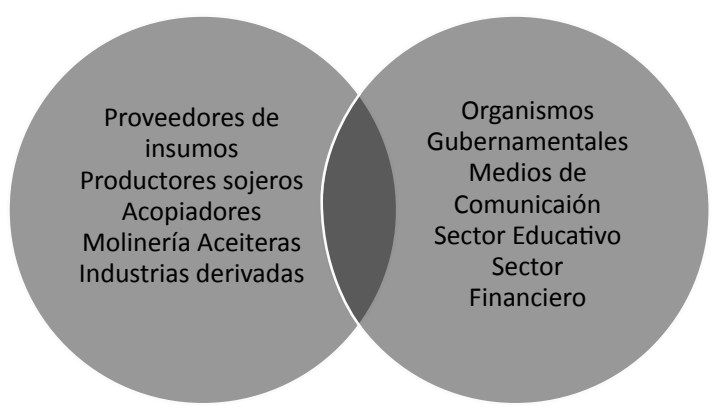

Sin embargo, debe señalarse que algunos sectores y empresas pueden decidir no participar de un clúster por diversas razones tales como la falta de confianza, entre otras.

En conclusión, dada la fortaleza del sistema productivo argentino, la provincia del Chaco se encuentra en condiciones de replicarlo impulsando explotaciones sojeras en el marco de un modelo de sustentabilidad (económica, social y ambiental) cohesionadas dentro un clúster productivo que cuente con la participación de productores, proveedores de insumos, instituciones gubernamentales y de investigación, ya que su naturaleza estratégica propiciará una gestión local superadora. 


\section{REFERENCIAS BIBLIOGRÁFICAS}

Cámara Argentina de Comercio -CAC-. (2013). Perfiles Exportadores provinciales junio 2013. Recuperado de app.kontakti.com.ar/data/mimgs/26_Perfiles\%2oExportadores\%20 Provinciales\%202013\%20F.pdf

Capitanich, J. M. (2011). Chaco: El secreto de la Argentina. Un ensayo histórico, político, económico y social. Resistencia, Argentina: Librería De La Paz.

Castagna, A. I., Pellegrini, J. L. \& Woelflin, M. L. (Dirs.) (2011). El nuevo sistema agro exportador en el sur de Santa Fe. Rosario, Argentina: UNR Editora.

Cristina, D., \& Bulgarelli, V. (2013). El valor de la producción de soja de 2013 rondaría los U\$S 26.000 millones, el máximo valor en los últimos 12 años (Informe económico $\mathrm{N}^{\circ}$ 213). Recuperado del sitio de internet del Instituto Argentino de Análisis Fiscal (IARAF): www.iaraf.org/informeszona.asp?busq $=\&$ buscar $=\& \mathrm{f}=\& 0=\& \mathrm{p}=7 \& \mathrm{r}=10$

Galeano, J.P. (2008, agosto). Comercialización de granos: etapas de la comercialización. Conferencia presentada en la Bolsa de Comercio de Rosario, Rosario.

Giancola, S., Salvador, M. L., Covacevich, M. e Iturrioz, G. (2009). Análisis de la cadena de valor de la soja en la Argentina. Estudios socioeconómicos de los sistemas agroalimentarios y agroindustriales, (3), 1-117.

Hirschman, A. (1961). La estrategia del desarrollo económico ( $2^{\circ}$ ed.). México: Fondo de Cultura Económica.

Martínez Dougnac, G. (s.f.). Apuntes acerca de la historia de la soja en la Argentina. Elementos para delinear experiencias comparadas. Recuperado del sitio de la Universidad de Buenos Aires, servicio de biblioteca digital: www.econ.uba.ar/www/servicios/Biblioteca/ bibliotecadigital/Indice\%20alfabetico/archivos/Apuntes\%2ocerca\%2ode\%20la\%20 historia\%20de\%2ola\%20soja\%2oen\%2ola\%20Argentina.\%2oElementos\%2opara\%20 delinear\%20experiencias\%2ocomparadas.pdf

Más, D. (1998). Etapa primaria de la comercialización de granos. Lecturas sobre comercialización de granos, 1, 12-23. 
Mitnik, F. (Coord.) (2011). Desarrollo de cadenas productivas, clusters y redes empresariales: Herramientas para el Desarrollo Territorial. Fondo Multilateral de Inversiones, Banco Interamericano de Desarrollo, Agencia para el Desarrollo Económico de Córdoba. Recuperado de www.adec.org.ar/biblioteca/libros/20

Morales, M. (2012). La cadena de valor del sector porcino en la Provincia del Chaco. (Tesis inédita del Posgrado de Especialización en Gestión de la Empresa Agropecuaria). Universidad Nacional del Nordeste, Resistencia, Chaco, Argentina.

Porter, M. (1998). On Competition (5a. ed.). Boston, MA: The Harvard Business Review book series.

Rapoport, M. (2008). Historia económica, política y social de la Argentina (1880-2003) (2a. ed.). Buenos Aires, Argentina: Emecé.

Sturzenegger, F. (2013). Yo no me quiero ir: Claves y razones para apostar por la Argentina. Planeta. Recuperado de www.amazon.com/Yo-quiero-razones-apostar-Argentinaebook/dp/BooDGoM4EG

\section{CURRICULUM VITAE}

\section{Carlos Harold Gabriel Lucas}

Estudiante del cuarto año de la carrera de Licenciatura en Administración en la Facultad de Ciencias económicas de la Universidad Nacional del Nordeste. Ayudante alumno en la Asignatura Contabilidad para Administradores de la carrera de Licenciatura en Administración en la Facultad de Ciencias Económicas de la Universidad Nacional del Nordeste (2012-2013). Becario de investigación de la Secretaría General de Ciencia y Técnica de la UNNE en el proyecto "Eficiencia Colectiva en la instalación de clúster en la base de la cadena de valor en la producción de soja en la Provincia del Chaco" (2013). $1^{\circ}$ Premio del concurso "Mejor Trabajo presentado por Becarios”. UNNE. Junio de 2014; Mención Especial en las Olimpiadas de Geografía de la República Argentina Año 2007. C.E.P. N 67 “Gral. Manuel Belgrano”. Junio de 2007. carloslucas@outlook.com 